\title{
Natural Frequencies and Acoustic Radiation Mode Amplitudes of Laminated Composite Plates Based on the Layerwise FEM
}

\author{
JinWu Wu and LingZhi Huang \\ School of Aircraft Engineering, Nanchang Hangkong University, Nanchang, People's Republic of China
}

\begin{abstract}
(Received 21 April 2012; revised 27 December 2012; accepted 7 February 2013)
In this paper, the natural frequencies and acoustic radiation mode amplitudes of laminated composite plates are studied. The layerwise finite element model is imposed to determine the natural frequencies and velocity distributions of laminated composite plates. The amplitude of the laminated composite plates are then discussed based on the acoustic radiation mode, the effects of the panel orientation angle, the elastic modulus ratio, the widthdepth ratio, and the damping ratio on the first acoustic radiation mode. A sixteen-layer laminated plate was used as an example, and the numerical simulations and experimental results show that the natural frequencies of the laminated composite plate can be analysed accurately using the proposed model. Furthermore, it is found that the effects of the panel orientation angle and width-depth ratio on the acoustic radiation mode amplitude of the laminated composite plates are significant.
\end{abstract}

\section{INTRODUCTION}

Laminated composite plates have been widely used in aerospace vehicles, maritime carriers, and wind turbine blades, where high strength, high stiffness, and low weight are important properties. ${ }^{1}$ When these composite structures are used in dynamic environments, vibration control and noise reduction become of great technical significance. The vibration of laminated composite structures is generally in low-frequency ranges. Thus, noise reduction of laminated composite structures in low-frequency ranges is very important. Although active vibration control could be used to decrease structural noise, ${ }^{1}$ low noise design is the most reliable means of reducing radiated noise. The most suitable function of a low noise design is in sound radiation power. ${ }^{2,3}$ A low noise design optimisation is considered with the goal of minimising the total sound power of the structure. The sound radiation power is related to the characteristics of the structure. ${ }^{2-4}$ Therefore, it is important to study the relation of the laminated composite structure parameters to the sound radiation power in low-frequency ranges.

For planar radiators, it is well known that the low-frequency sound radiation is directly related to the velocity distribution of the structure's surface. Different velocity distributions have different contributions to the radiation sound power. Velocity distributions corresponding to certain mode shapes are more efficient radiators. It has been widely accepted that structural acoustic problems can be analysed based on so-called radiation modes. ${ }^{1}$ Radiation modes are sets of independent radiating velocity distributions. The dominant radiation modes are the first few order modes at low-frequency ranges. Controlling the first few order radiation modes' amplitudes has been shown to reduce the total sound power efficiently in low-frequency ranges. $1,5,6$

For laminated composite plates, the dynamic response of the structures must first be analysed in order to study the first few order radiation modes' amplitudes of the plates in lowfrequency ranges. Laminated plate theories are essential to providing an accurate analysis of laminated composite plates, and a variety of laminated plate theories have been developed and reported in the literature. A review of the various equivalent single layer and layerwise laminated plate theories can be found in Reddy's work. ${ }^{7}$ Because of their complex behaviour in the analysis of laminated composite plates, some technical aspects must be taken into consideration. For example, the classical laminate plate theory (CLPT) is based on the Kirchhoff plate theory. It is the simplest theory, but the shear deformation effects are neglected. ${ }^{8}$ Furthermore, it results in an underestimate of the deflection and an overestimate of the natural frequencies. The first and higher order shear deformation theories are improvements over classical theories. For the first and higher order shear deformation theories, transverse shear deformation through the thickness of the structure is not ignored. ${ }^{9}$

Another aspect in the analysis of composite structures is the existence of couplings among stretching, shearing, bending, and twisting. These couplings can significantly change the response of composite structures and need to be considered. The layerwise lamination theory assumes a displacement representation formula in each layer. ${ }^{10}$ The layerwise finite element theory can be seen as a three-dimensional theory. The assumed layerwise displacement field uses a linear Lagrange polynomial approximation for the thickness of each lamina and a constant transverse displacement for the entire thickness. The interlaminar stresses can be predicted accurately, and the layerwise finite element theory can better adapt to the combination of boundary conditions. In this paper, the dynamic responses of the laminated composite plates are examined, based on a layerwise finite element theory.

In recent years, there has been much research on the vibration and acoustical characteristics of laminated composite plates. For example, Li et al. ${ }^{11,12}$ deal with the structural vibration and acoustic radiation of a fluid-loaded laminated plate based on the first order shear deformation theory (FSDT) and the classical Kirchhoff-Love thin plate theory. Cao et al. ${ }^{13}$ 